\title{
Benzodiazepine receptor agonist dispensations in Alberta: a population-based descriptive study
}

\author{
Daniala L. Weir MSc, Salim Samanani MD, Fizza Gilani BScPharm, Ed Jess BA, Dean T. Eurich PhD
}

Abstract

Background: There is increasing concern over the use of benzodiazepine receptor agonists (BZRAs). The objective of this study was to describe BZRA dispensations in the province of Alberta in 2015 according to age, sex and appropriateness.

Methods: A population-based descriptive study of people 10 years of age or older with at least 1 BZRA dispensation in Alberta, Canada, between Jan. 1 and Dec. 31, 2015, was conducted. Prevalence of BZRA use, characteristics of BZRAs dispensations, use at the individual level and appropriateness were determined.

Results: A total of 372870 people received 2463585 BZRA dispensations in Alberta in 2015. Prevalence of use at the population level was $10 \%$ overall, increased with age $(p$ value for trend $<0.001)$ and was consistently highest among females. Twenty percent of patients used both Z-drugs and benzodiazepines. BZRA users had an average of 7 dispensations (standard deviation [SD] 20), 137 days of use overall (SD 123) and a maximum period of consecutive use of 90 days (SD 95). Days of consecutive use were highest among those aged 65 years or older (126 d). A total of 62795 (17\%) people used more than 1 distinct BZRA ingredient concurrently and $10 \%$ had 3 or more distinct prescribers.

Interpretation: The prevalence of BZRA use was high and a substantial proportion of use appeared to be potentially inappropriate. This study supports the need for continued monitoring for the prescribing and use of these medications at the population level.

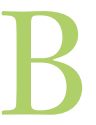

enzodiazepine receptor agonists (BRZAs), including benzodiazepines and Z-drugs, are primarily indicated for the treatment of anxiety or sleep disorders; clinical practice guidelines recommend treatment for short durations (less than $4 \mathrm{wk}$ ) in adults (age 20-64 yr). Use of BZRAs outside of these recommendations is considered "potentially inappropriate" given the increased potential for adverse effects. ${ }^{1-3}$ Extended use of BZRAs can lead to depression, memory loss, addiction and even death. ${ }^{4-6}$ A number of evidence-based guidelines that have identified potentially inappropriate medications in patients 65 years of age or older recommend against the use of BZRAs in this patient population because of the increased risk of delirium, falls, hip fractures, cognitive impairments and avoidable emergency department visits. ${ }^{7-9}$ In addition, sedative hypnotics account for a substantial number of avoidable emergency department visits and hospital admissions in older adults. ${ }^{9}$

As a result, national and provincial initiatives in Canada have aimed at reducing inappropriate BZRA prescribing as well as decreasing the potential for harm. ${ }^{10-12}$ However, studies evaluating trends in inappropriate use and prescribing have been limited and do not reflect the expected outcomes of these initiatives. In British Columbia, nearly 1 in 10 older adults filled prescriptions for a benzodiazepine or benzodiazepine-like sedative with more than a 90-day supply in $2013 .{ }^{13}$ In this population, these medications were used in the long term more frequently than any other potentially inappropriate medications..$^{14}$ Between 1996 and 2012, the incidence of benzodiazepine use decreased considerably among older adults in Manitoba, but the use of Z-drugs increased and the prevalence of benzodiazepine use remained higher in older adults than in any other age group. ${ }^{15}$ Moreover, dose escalations were common in those under the age of 44 years from 1996 to $2008 .{ }^{16}$ In Ontario, benzodiazepine prescribing decreased in those 65 years of age or older between 2002 and 2016, but prevalence remained high (around 12\% of older adults). ${ }^{17,18}$ However, as a result of new legislation implemented in 2011, inappropriate benzodiazepine prescribing decreased by $50 \%{ }^{11}$ Additionally, a cross-sectional study of

Competing interests: Salim Samanani reports receiving a grant from the College of Physicians \& Surgeons of Alberta, during the conduct of the study. No other competing interests were declared.

This article has been peer reviewed.

Correspondence to: Dean Eurich, deurich@ualberta.ca

CMAJ Open 2018. DOI:10.9778/cmajo.20180121 
benzodiazepines dispensed to Canadians 65 years of age or older in 2013 found that prevalence varied substantially from province to province; Alberta was found to have one of the lowest prescribing rates (well below the national average) while Quebec had the highest. ${ }^{19}$ A study of benzodiazepine use in all Quebec residents over the age of 65 years found that the mean duration of uninterrupted use was 80 days, and $8 \%$ of seniors in that province filled 2 or more prescriptions concurrently. ${ }^{20}$

Given the widespread and potentially inappropriate use of these medications across Canada, the variations in prescribing observed between provinces and the fact that Alberta implemented strong legislation around the prescribing of BZRAs in 2015, the objective of this study was to describe dispensations of BRZAs for all people in the province of Alberta in 2015.

\section{Methods}

\section{Study population}

Our study cohort included people 10 years of age or older who were dispensed at least 1 BZRA drug in an Alberta community pharmacy between Jan. 1 and Dec. 31, 2015.

\section{Data sources}

Data from Alberta's Pharmaceutical Information Network $(\mathrm{PIN})^{21}$ were used for the analysis. PIN data include all dispensing records from community pharmacies, thus providing comprehensive data on all medication dispensations (from all prescriber types) occurring in the province outside of the hospital setting. Information on the dispensed medication (drug identification number [DIN], dispensing date, quantity, days supply, strength, medication name, instructions), patient information (unique patient identifier, age, sex) and prescriber information (licence number, prescriber type) were available. The validity of the days supply variable for each dispensation was evaluated to ensure it fell within a plausible clinical range on the basis of the defined daily dose for a single dispensation; less than $0.01 \%$ of the days supply values were deemed to be outside of this range and a new days supply value was imputed on the basis of a person's historical average for a particular ingredient. All unique identifiers (patient, prescriber) were anonymized for the purposes of this analysis. Information on the size and age/sex distribution of the Alberta population in 2015 was obtained from Alberta Vital Statistics. ${ }^{22}$

\section{Study measures}

All BZRAs dispensations were identified in the PIN data and classified according to ingredient (alprazolam, bromazepam, chlordiazepoxide, clobazam, clonazepam, diazepam, flurazepam, lorazepam, midazolam, nitrazepam, oxazepam, temazepam, triazolam, zolpidem and zopiclone) and drug class (benzodiazepines or Z-drugs). For each BZRA dispensation a patient received, the number of defined daily doses was calculated on the basis of the strength, quantity and days supply as well as the defined daily dose for each ingredient. ${ }^{23}$ Although we were not able to directly observe utilization of these medications by patients, we considered "use" as any day on which a patient had a supply of BZRA medication on hand on the basis of the date and days supply of each dispensation. Using information on the ingredient dispensed, dispensing date, days supply and quantity for each BZRA dispensation, we calculated a binary variable for each BZRA ingredient on each day of follow-up for an individual patient, to determine whether they had a supply of that particular ingredient on hand on a given day. We were then able to calcualte how many unique BZRA ingredients patients were using (i.e., whether they were concurrently using 2 unique ingredients at the same time), as well as the number of days a patient had a supply of BZRAs on hand. A consecutive period of BZRA use was any period of time in which a patient had an uninterrupted supply of BZRA medications; if a patient had at least a 3-day gap in their supply, this indicated the end of a consecutive period of use. We also conducted a sensitivity analysis to determine the impact that extending this gap to 7 days would have on our findings related to days of consecutive use.

Our choice of indicators of potentially inappropriate use was informed by Canadian clinical practice guidelines for anxiety and insomnia, the criteria of Choosing Wisely Canada and the Beers and STOPP (Screening Tool of Older Persons' Prescriptions) criteria ${ }^{7,10,24}$ and defined as follows: (a) use of BZRAs for more than 30 consecutive days, (b) use of more than 1 BZRA ingredient concurrently and (c) the prescription of BZRAs to the same patient by multiple prescribers.

\section{Statistical analyses}

A number of descriptive analyses were conducted. First, the prevalence of BZRA use per 100 residents in 2015 was determined for 10-year age groups and by sex. Characteristics of BZRA dispensations, stratified by drug class (benzodiazepines v. Z-drugs), were determined according to prescriber type, days supply and number of defined daily doses. Individual-level indicators of potentially inappropriate BZRA use and prescribing were calculated by age category (youth [10-19 yr], adults [20-64 yr], older adults [ $\geq 65 \mathrm{yr}]$ ) and sex. The top 5\% of users for each patient-level indicator were also determined. We calculated $p$ values for trend according to age and sex for a number of indicators, but only for descriptive purposes; statistical testing of a specific hypothesis was not conducted. All analyses were performed using Stata/SE version 14.0 (Stata Corp.).

\section{Ethics approval}

Ethics approval for this study was provided by the University of Alberta (ethics approval no. Pro00083807).

\section{Results}

Overall, 372870 patients received 2463585 BZRA dispensations in 2015 from 11944 distinct prescribers. The majority of users were female (63\%) and the mean age was 54 years (standard deviation [SD] 21). In this study, 31\% of patients were dispensed benzodiazepines alone, 49\% were dispensed Z-drugs alone and 20\% were dispensed both Z-drugs and benzodiazepines. On average, patients received 7 dispensations (SD 20) for a total of 137 days of use (SD 123) during the year-long study period. 
Ten percent of the Alberta population (over $10 \mathrm{yr}$ of age) was dispensed a BZRA in 2015 and the prevalence of use steadily increased with age ( $p$ value for trend $<0.001$ ) (Figure 1 ). While only 1 in 100 patients aged $10-19$ years were using these medications, this proportion increased to 14 per 100 in patients aged 50-59 years and rose to 30 per 100 in those aged 90 years or older. Prevalence of BZRA use was higher among females than among males for each age category (Figure 1). Overall, $13 \%$ of females were dispensed a BZRA in 2015 compared with $8 \%$ of males $(p<0.001)$ (Appendix 1, available at www.cmajopen.ca/content/6/4/E678/suppl/DC1).

When we evaluated the characteristics of BZRA dispensations by drug class, we found that $44 \%$ were $Z$-drugs and $56 \%$ were benzodiazepines. Characteristics were similar between drug classes. The average days supply for any BZRA dispensation was 27 days (SD 28): 23 days (SD 25) for benzodiazepines and 33 days (SD 30) for Z-drugs. Seventeen percent of all dispensations (12\% of benzodiazepines and $22 \%$ of $Z$-drugs) had a supply over 30 days. The average number of defined daily doses for BZRA dispensations overall was 1.0 (SD 0.7); however, approximately $26 \%$ of dispensations were for 2 or more defined daily doses (Table 1).

When we evaluated potentially inappropriate BZRA use at the individual level according to age and sex, we found that characteristics were similar between males and females, but some indicators varied by age (Table 2). The average maximum period of consecutive use was 90 days (SD 95) overall and we observed increasing periods of consecutive use with increasing age ( $p$ value for trend $<0.001)$; while youth aged 10-19 years had an average maximum period of consecutive use of 31 days (SD 48), this increased to 126 days (SD 102) in patients aged 65 years or older. Additionally, we found that $71 \%$ of patients had a maximum period of consecutive use greater than 30 days. Most patients used 1 BZRA ingredient at a time; however, $17 \%$ of patients had 2 or more ingredients on hand concurrently. Concurrent use of multiple ingredients was relatively similar across age categories. Lastly, we found that most patients were prescribed medications by 1 or 2 prescribers (mean number of distinct prescribers 1.5, SD 1.0); however, $4 \%$ had 4 or more prescribers. Patients aged 20-64 years had more prescribers than youth and older adults $(p<0.001)$.

When we examined the top $5 \%$ of users for each patientlevel indicator of potentially inappropriate BZRA use, we found that these individuals had 22 or more dispensations in the year, 3 or more prescribers, 2 or more unique BZRA ingredients dispensed concurrently and 347 or more days of consecutive use.

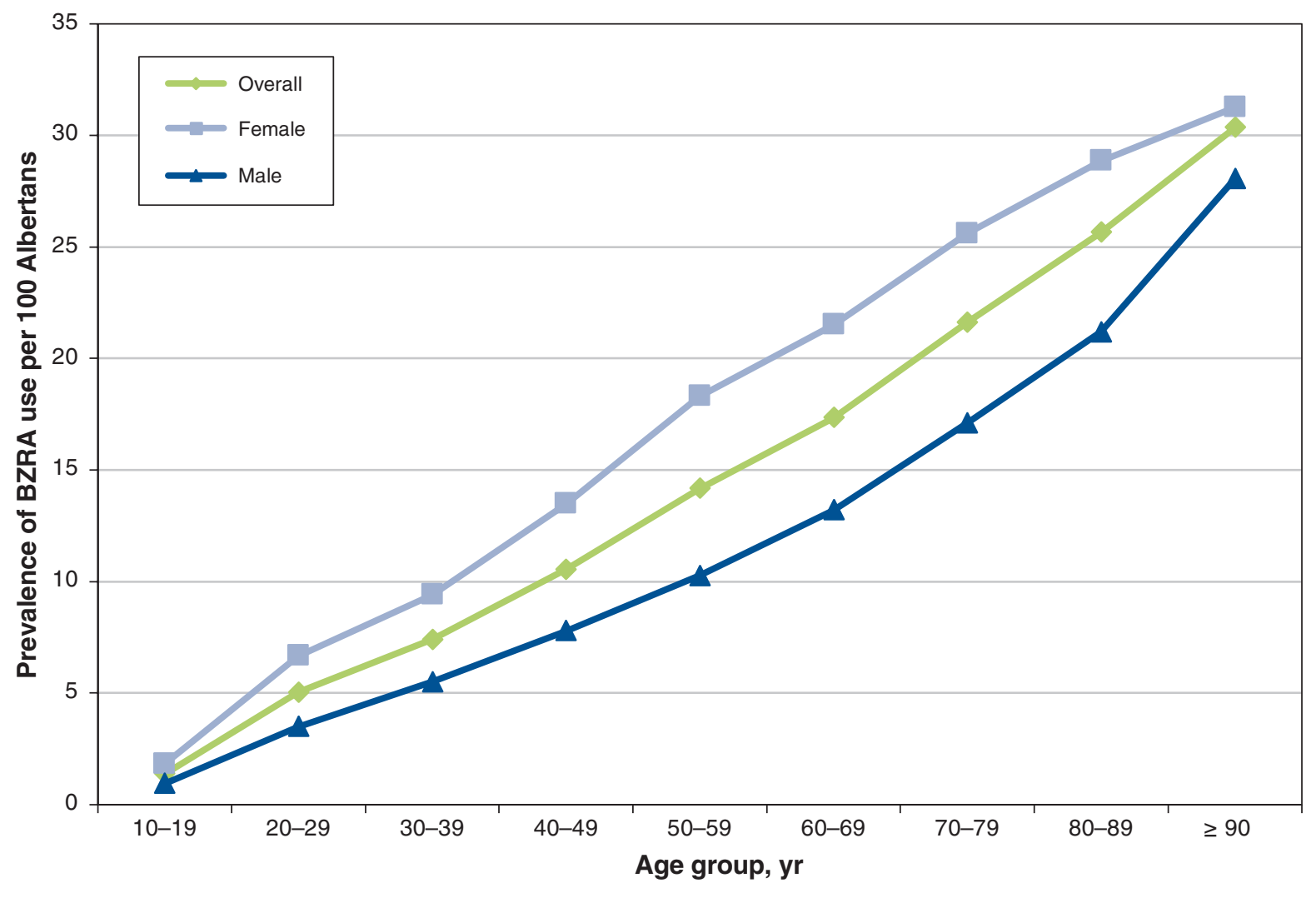

Figure 1: Prevalence of benzodiazepine receptor agonist (BZRA) use in Alberta by 10-year age group and sex in 2015. 


\begin{tabular}{|c|c|c|c|}
\hline Characteristic & $\begin{array}{l}\text { All BZRAs, } \\
\text { no. }(\%)^{*} \\
n=2463585\end{array}$ & $\begin{array}{l}\text { Benzodiazepines, } \\
\text { no. }(\%)^{*} \\
n=1391169\end{array}$ & $\begin{array}{l}\text { Z-drugs, } \\
\text { no. }(\%)^{*} \\
n=1072416\end{array}$ \\
\hline \multicolumn{4}{|l|}{ Prescriber type } \\
\hline Physician & 2392459 (97.1) & $1363278(98.0)$ & $1029181(96.0)$ \\
\hline Pharmacist & $21766(0.9)$ & $327(0.02)$ & $21439(2.0)$ \\
\hline Dentist & $8051(0.3)$ & $1829(0.6)$ & $222(0.02)$ \\
\hline Nurse practitioner & $9694(0.4)$ & $3598(0.3)$ & $6096(0.6)$ \\
\hline Other & $70(-)$ & $47(-)$ & $23(-)$ \\
\hline Unknown & $31545(1.3)$ & $16090(1.2)$ & $15455(1.4)$ \\
\hline \multicolumn{4}{|l|}{ Days supply† } \\
\hline Mean \pm SD & $27 \pm 28$ & $23 \pm 25$ & $33 \pm 30$ \\
\hline $1-7$ & $819694(33.3)$ & $532182(38.3)$ & $287512(26.8)$ \\
\hline $8-14$ & $231465(9.4)$ & $158762(11.4)$ & $72703(6.8)$ \\
\hline $15-30$ & $1010202(41.1)$ & $535874(38.5)$ & $474328(44.2)$ \\
\hline $31-60$ & $140901(5.7)$ & $64215(4.6)$ & $76686(7.2)$ \\
\hline $61-90$ & $139714(5.7)$ & $53722(3.9)$ & $85992(8.0)$ \\
\hline$\geq 90$ & 121609 (4.9) & 46414 (3.3) & $75195(7.0)$ \\
\hline \multicolumn{4}{|c|}{ No. of defined daily doses $† \ddagger$} \\
\hline Mean \pm SD & $1.0 \pm 0.7$ & $0.8 \pm 0.8$ & $1.2 \pm 0.6$ \\
\hline 1 & 1814149 (73.6) & 1016813 (73.1) & $797336(74.4)$ \\
\hline 2 & $617767(25.1)$ & $347875(25.0)$ & 269892 (25.2) \\
\hline$\geq 3$ & 31669 (1.3) & 26481 (1.9) & $5188(0.5)$ \\
\hline \multicolumn{4}{|c|}{$\begin{array}{l}\text { Note: } S D=\text { standard deviation. } \\
\text { *Except where indicated otherwise. } \\
\text { †Standardized differences between benzodiazepines and Z-drugs for mean days supply and mean no. of defined daily } \\
\text { doses are } 0.36 \mathrm{~d} \text { and } 0.57 \text { defined daily doses, respectively. } \\
\ddagger \text { No. of defined daily doses was rounded to the nearest whole number. }\end{array}$} \\
\hline
\end{tabular}

\section{Interpretation}

Overall, we found that 1 in 10 people over 10 years of age were dispensed a BRZA in 2015, with the highest prevalence of use among females and patients 65 years of age or older. Not only was prevalence of use high, the majority of individuals used these medications for more than 30 consecutive days. A substantial proportion of patients also used more than 1 distinct BZRA ingredient concurrently and had multiple prescribers.

Although some patients may need to stay on BZRAs for specific clinical reasons, most should use BZRAs for a short period of time only. Substantial efforts have been made by a number of organizations, including the Canadian Deprescribing Network, to provide physicians and patients with tools to support them in reducing inappropriate medication use and to provide specific guidance for deprescribing benzodiazepines. ${ }^{25}$ Similarly, a cluster-randomized trial (Eliminating Medications Through Patient Ownership of End Results) demonstrated a $27 \%$ benzodiazepine discontinuation rate with an intervention to engage older adults in discussing the harms of benzodiazepine use with their physician and/or pharmacists, compared with a $5 \%$ reduction in the control group. ${ }^{26}$ Thus, effective tools do exist to taper and discontinue use of these medications in older adults, especially for insomnia. It should be acknowledged, however, that for many people it is very difficult to stop taking BZRAs. ${ }^{27,28}$ Informing patients about the potential for addiction and difficulty stopping these medications is probably warranted upon initiation of BZRAs. In Alberta, the College of Physicians \& Surgeons supports the appropriate prescribing of BZRAs by linking members to resources, education and tools available through its website. Also, prescribing reports are provided to physicians that identify their patients who received BZRAs above certain thresholds (i.e., age $>65 \mathrm{yr}$, dose $>2$ defined daily doses, multiple BZRA ingredients and combination with opioids). These reports are reflective tools to help improve prescribing awareness and build on data from Alberta's prescription monitoring program. ${ }^{12}$ At the federal level, Health Canada issued guidance on the dosing of Z-drugs in 2014, recommending that the initiation dose of zopiclone be reduced to $3.75 \mathrm{mg}$ daily and that the maximum daily dose of zopiclone be reduced to $7.5 \mathrm{mg}$ in healthy people and to $5 \mathrm{mg}$ in elderly people or 


\begin{tabular}{|c|c|c|c|c|c|c|}
\hline & \multirow[b]{2}{*}{$\begin{array}{c}\text { Overall } \\
n=372870\end{array}$} & \multicolumn{3}{|c|}{ Age category, yr, no. $(\%)^{\star}$} & \multicolumn{2}{|c|}{ Sex, no. $(\%)^{*}$} \\
\hline & & $\begin{array}{c}10-19 \\
n=6550\end{array}$ & $\begin{array}{c}20-64 \\
n=260600\end{array}$ & $\begin{array}{c}\geq 65 \\
n=105720\end{array}$ & $\begin{array}{c}\text { Female } \\
n=234140\end{array}$ & $\begin{array}{c}\text { Male } \\
n=138725\end{array}$ \\
\hline \multicolumn{7}{|c|}{ Maximum period of consecutive use, $d$} \\
\hline Mean \pm SD & $90 \pm 95$ & $31 \pm 48$ & $77 \pm 89$ & $126 \pm 102$ & $91 \pm 96$ & $90 \pm 94$ \\
\hline$\leq 30$ & $106603(28.6)$ & $4400(67.2)$ & $86863(33.3)$ & $15340(14.5)$ & $67663(28.9)$ & $38937(28.1)$ \\
\hline $31-90$ & $124090(33.3)$ & $1555(23.7)$ & $93894(36.0)$ & $28641(27.1)$ & 76949 (32.9) & $47140(34.0)$ \\
\hline $91-120$ & $44575(12.0)$ & $228(3.5)$ & $25073(9.6)$ & $19274(18.2)$ & $28199(12.0)$ & $16376(11.8)$ \\
\hline$\geq 121$ & $97602(26.2)$ & $267(5.6)$ & $54770(21.0)$ & 42465 (40.2) & $61329(26.2)$ & $36272(26.2)$ \\
\hline \multicolumn{7}{|c|}{ Maximum no. of unique ingredients used concurrently } \\
\hline 1 & $310075(83.2)$ & $6040(92.2)$ & $214385(82.3)$ & $89650(84.8)$ & $193179(82.5)$ & $116891(84.3)$ \\
\hline 2 & $55072(14.8)$ & $482(7.4)$ & 39899 (15.3) & 14691 (13.9) & 35847 (15.3) & 19225 (13.9) \\
\hline$\geq 3$ & $7723(2.1)$ & $28(0.4)$ & $6316(2.4)$ & $1379(1.3)$ & $5115(2.2)$ & 2609 (1.9) \\
\hline \multicolumn{7}{|c|}{ No. of distinct prescribers } \\
\hline Mean \pm SD & $1.5 \pm 1.0$ & $1.2 \pm 0.6$ & $1.5 \pm 1.1$ & $1.4 \pm 0.8$ & $1.5 \pm 1.0$ & $1.5 \pm 10$ \\
\hline 1 & $265620(71.2)$ & $5413(82.6)$ & $184864(70.9)$ & 75343 (71.3) & $167950(71.7)$ & $97666(70.4)$ \\
\hline 2 & 70300 (18.9) & 847 (12.9) & $47981(18.4)$ & 21472 (20.3) & $43810(18.7)$ & 26489 (19.1) \\
\hline 3 & $22210(6.0)$ & $193(3.0)$ & $15784(6.1)$ & $6233(5.9)$ & $13645(5.8)$ & $8656(6.2)$ \\
\hline$\geq 4$ & $14740(4.0)$ & $97(1.5)$ & 11971 (4.6) & $267(2.5)$ & $8735(3.7)$ & 6005 (4.3) \\
\hline
\end{tabular}

those with renal or liver failure. However, a recent review of benzodiazepine use at the population level in Canada pointed out that national prescription guidelines for benzodiazepines are lacking and that there is a clear need for reducing inappropriate use and related harm through improved evidence-based practices as well as monitoring and control. ${ }^{29}$

Prevalence of BZRA use in Alberta was similar to that in studies conducted in BC and Manitoba. A population-based examination of BZRA dispensations in Manitoba found that the prevalence of BZRA use for 2012 was about 1 user per 10 residents, ${ }^{15}$ while in $\mathrm{BC} 8.4 \%$ of all residents were users of benzodiazepines. Moreover, in BC, $41 \%$ of all benzodiazepine users were defined as long-term users (i.e., they had at least 100 days supply in the year). ${ }^{30}$ This was similar to our finding that $44 \%$ of Alberta users had a supply of BZRAs for at least 120 days of the year. However, our results differed from those of a study of older Quebec residents in that fewer residents filled concurrent prescriptions in Quebec than in Alberta. ${ }^{20}$ Additionally, a recent study of $\mathrm{BC}$ residents over the age of 65 years found that women were more likely to receive inappropriate benzodiazepine prescriptions than men; however, we did not find differences in measures of potentially inappropriate use according to sex. Moreover, Black and colleagues observed substantial differences between provinces in the prevalence of use of BZRAs by older adults. The authors suggest that since access to BZRAs through public drug programs is generally similar across most provinces, utilization differences are probably driven by factors unrelated to formulary listing, such as stricter policies around prescribing of these medications and mandated monthly clinical reviews of continued use. ${ }^{19}$

Our study was similar to others in that prevalence of use was higher among females than among males. ${ }^{13,15}$ Although additional studies are required to explore sex differences in prevalence of use, these differences may be explained in part by biological differences in the prevalence of conditions that are indications for BZRA treatment or differences in health services use such as the frequency of visits to health care providers. This population-based study had a number of important strengths, including the analysis of data that provided a full picture of all BZRA dispensations by community pharmacies in the province. Additionally, measures of potentially inappropriate use were based on clinical practice guidelines for the treatment of insomnia and anxiety, as well as published criteria to identify potentially inappropriate prescribing. Given that use of these medications for more than 4 weeks at a time substantially increases the risk for adverse effects, it was important to gain a better understanding of this aspect of prescribing and utilization in Alberta. Our results also provide evidence of the potential mechanisms underlying inappropriate use of BZRAs that could be applicable to other Canadian provinces, pointing to future areas of potential focus for provincial prescription drug monitoring programs or quality improvement initiatives. 


\section{Limitations}

There are a number of limitations to keep in mind when interpreting the results of this study. First, we were only able to analyze dispensed medications; we do not know whether or not patients actually took them. While longterm use can lead to tolerance, dependence and addiction, ${ }^{31}$ making it likely that patients were actually taking dispensed medications, BZRAs are also known to carry a diversion risk. Moreover, although BZRAs are typically prescribed on an "as needed" basis, if a patient were not actually using the prescription dispensed to them the length of time between dispensations would probably increase, decreasing the likelihood of prolonged use. Therefore, if a patient has 2 different ingredients on hand at once or visits the pharmacy to refill their prescription early, they are probably actually using their medications. This being said, we still cannot measure medication use directly by analyzing dispensing data, and this is a clear limitation. Second, we can only study potentially inappropriate utilization in this study, not truly inappropriate use, as we did not have access to detailed clinical information or the indication for BZRA use. For most adults, the primary indications are for sleep and anxiety disorders, but these drugs can also be used in the treatment of epilepsy, where chronic use may be entirely appropriate. However, at the population level, we would expect only a small proportion of individuals to use BZRAs for epilepsy $(0.4 \%$ of the Canadian population had epilepsy in 2012). ${ }^{32}$ Additionally, clinical practice guidelines for the appropriate use of BZRAs typically apply to the adult population alone. We have included people younger than 18 years of age in our analysis as an increasing body of literature suggests that BZRAs are increasingly being abused by this age group. However, we acknowledge we are limited in our ability to draw conclusions about appropriate use in these individuals. Although the PIN database provides the first opportunity to study population-based community dispensing in Alberta, it has not been used extensively for research purposes to date. Therefore, validation studies specifically evaluating the accuracy of the data set have yet to be published. Last, we did not investigate the impact of potentially inappropriate medication use on adverse health outcomes such as emergency department visits, hospital admisssions or falls; conducting this type of study would be an important next step in determining the burden associated with potentially inappropriate use of BZRAs.

\section{Conclusion}

The prevalence of BZRA use was high in our study, and a substantial proportion of use appeared to be potentially inappropriate. Interventions aimed at educating patients on the potential harms of these medications are required, as are continuing education, monitoring and other regulatory interventions for prescribers. Prescribers must also continue to be provided with timely patient information to make treatment decisions, and continued monitoring of medication dispensing is critical to developing our understanding of this public health issue as it evolves over time.

\section{References}

1. Kennedy SH, Lam RW, Parikh SV, et al. Canadian Network for Mood and Anxiety Treatments (CANMAT) clinical guidelines for the management of major depressive disorder in adults. Introduction. 7 Affect Disord 2009; 117(Suppl 1):S1-2.

2. Katzman MA, Bleau P, Blier P, et al. Canadian clinical practice guidelines for the management of anxiety, posttraumatic stress and obsessive-compulsive disorders. BMC Psychiatry 2014;14(Suppl 1):S1.

3. Adult insomnia: diagnosis to management. Edmonton: Toward Optimized Practice Program: 2006; revised 2010. Available: http://www.topalbertadoctors.org/ download/439/insomnia_management_guideline.pdf (accessed 2018 May 3).

4. Tannenbaum C. Inappropriate benzodiazepine use in elderly patients and its reduction. 7 Psychiatry Neurosci 2015;40:E27-8.

5. Billioti de Gage S, Moride Y, Ducruet T, et al. Benzodiazepine use and risk of Alzheimer's disease: case-control study. BMF 2014;349:g5205.

6. Weich S, Pearce HL, Croft P, et al. Effect of anxiolytic and hypnotic drug prescriptions on mortality hazards: retrospective cohort study. BM7 2014; 348:g1996.

7. Davidoff AJ, Miller GE, Sarpong EM, et al. Prevalence of potentially inappropriate medication use in older adults using the 2012 Beers criteria. $7 \mathrm{Am}$ Geriatr Soc 2015;63:486-500

8. Dalleur O, Spinewine A, Henrard S, et al. Inappropriate prescribing and related hospital admissions in frail older persons according to the STOPP and START criteria. Drugs Aging 2012;29:829-37.

9. Hampton LM, Daubresse M, Chang H-Y, et al. Emergency department visits by adults for psychiatric medication adverse events. $7 A M A$ Psychiatry 2014;71:1006-14.

10. Five things physicians and patients should question. Markham (ON): Canadian Geriatrics Society; updated June 2017. Available: https://choosingwiselycanada. org/geriatrics/ (accessed 2017 Dec. 3).

11. Gomes T, Juurlink D, Yao Z, et al. Impact of legislation and a prescription monitoring program on the prevalence of potentially inappropriate prescriptions for monitored drugs in Ontario: a time series analysis. CMAZ Open 2014;2:E256-61.

12. TTP Alberta. Edmonton: College of Physicians \& Surgeons of Alberta. Available: www.cpsa.ca/triplicate-prescription-program-tpp/ (accessed 2017 Dec. 3).

13. Morgan SG, Weymann D. Patterns, predictors and persistence of chronic sedative use: a population-based observational study of older adults in British Columbia, Canada. Eur 7 Clin Pharmacol 2017;73:1001-8.

14. Morgan SG, Weymann D, Pratt B, et al. Sex differences in the risk of receiving potentially inappropriate prescriptions among older adults. Age Ageing 2016; 45:535-42.

15. Alessi-Severini S, Bolton JM, Enns MW, et al. Use of benzodiazepines and related drugs in Manitoba: a population-based study. CMAf Open 2014; 2:E208-16.

16. Alessi-Severini S, Bolton JM, Enns MW, et al. Sustained use of benzodiazepines and escalation to high doses in a Canadian population. Psychiatr Serv 2016;67:1012-8

17. Iaboni A, Reynolds KB, Bronskill S, et al. Changing patterns of sedative use over time in older adults in Ontario. Am 7 Geriatr Psychiatry 2016;24:S125. doi: 10.1016/j.jagp.2016.01.125.

18. Brett J, Maust DT, Bouck Z, et al. Benzodiazepine use in older adults in the United States, Ontario, and Australia from 2010 to 2016. 7 Am Geriatr Soc 2018;66:1180-5

19. Black CD, McCarthy L, Gomes T, et al. Interprovincial variation of psychotropic prescriptions dispensed to older Canadian adults. Can Geriatr 7 2018; 21:269-73.

20. Bartlett G, Abrahamowicz M, Tamblyn R, et al. Longitudinal patterns of new benzodiazepine use in the elderly. Pharmacoepidemiol Drug Saf 2004;13:669-82.

21. Pharmaceutical Information Network (PIN). Edmonton: Alberta Netcare Learning Centre. Available: www.albertanetcare.ca/learningcentre/PharmaceuticalInformation-Network.htm (accessed 2018 Nov. 1).

22. Data, statistics and publications. Government of Alberta. Available: www. alberta.ca/data-statistics-publications.aspx (accessed 2018 Nov. 1).

23. Hallas J. Drug utilization statistics for individual-level pharmacy dispensing data. Pharmacoepidemiol Drug Saf 2005;14:455-63.

24. Gallagher P, O'Mahony D. STOPP (Screening Tool of Older Persons' potentially inappropriate Prescriptions): application to acutely ill elderly patients and comparison with Beers' criteria. Age Ageing 2008;37:673-9.

25. Pottie K, Thompson W, Davies S, et al. Deprescribing benzodiazepine receptor agonists: evidence-based clinical practice guideline. Can Fam Physician 2018;64:339-51.

26. Tannenbaum C, Martin P, Tamblyn R, et al. Reduction of inappropriate benzodiazepine prescriptions among older adults through direct patient education: the EMPOWER cluster randomized trial. FAMA Intern Med 2014; 174:890-8.

27. Greenblatt DJ, Shader RI. Dependence, tolerance, and addiction to benzodiazepines: clinical and pharmacokinetic considerations. Drug Metab Rev 1978;8:13-28.

28. Weaver MF. Prescription sedative misuse and abuse. Yale 7 Biol Med 2015; $88: 247-56$. 
29. Murphy Y, Wilson E, Goldner EM, et al. Benzodiazepine use, misuse, and harm at the population level in Canada: a comprehensive narrative review of data and developments since 1995. Clin Drug Investig 2016;36:519-30.

30. Cunningham CM, Hanley GE, Morgan S. Patterns in the use of benzodiazepines in British Columbia: examining the impact of increasing research and guideline cautions against long-term use. Health Policy 2010;97:122-9.

31. O'brien CP. Benzodiazepine use, abuse, and dependence. 7 Clin Psychiatry 2005;66(Suppl 2):28-33.

32. Gilmour H, Ramage-Morin P, Wong SL. Epilepsy in Canada: prevalence and impact. Health Rep 2016;27:24-30.

Affiliations: Clinical and Health Informatics Research Group (Weir), Faculty of Medicine and Department of Epidemiology, Biostatistics and Occupational Health (Weir), McGill University, Montréal, Que.; Okaki Health Intelligence (Samanani), Calgary, Alta.; College of Physicians \& Surgeons of Alberta (Gilani, Jess); School of Public Health (Eurich), University of Alberta; Alliance for Canadian Health Outcomes Research in Diabetes (Eurich), Edmonton, Alta.
Contributors: All authors conceived and designed the study. Salim Samanani and Ed Jess acquired the data. Daniala Weir did the statistical analyses. All authors analyzed and interpreted the data. Daniala Weir wrote the manuscript, and all authors critically revised it. All authors approved the final version of the manuscript and agree to act as guarantors of the work.

Acknowledgements: This study is based in part on data provided by Alberta Health and was commissioned by the College of Physicians \& Surgeons of Alberta. The interpretation and conclusions contained herein are those of the researchers and do not necessarily represent the views of the Government of Alberta or the College of Physicians \& Surgeons of Alberta. Neither the Government of Alberta nor Alberta Health expresses any opinion in relation to this study.

Supplemental information: For reviewer comments and the original submission of this manuscript, please see www.cmajopen.ca/content/6/4/ E678/suppl/DC1. 